\title{
Tamanho Mínimo de Amostra de Plantas e Frutos do Algodoeiro para Monitoramento das Populações de Anthonomus grandis Boh. (Coleoptera: Curculionidae)
}

\author{
Ivo Pierozzi Jr. ${ }^{1}$, Sidnei Ragazzi² e Mohamed E.M. Habib ${ }^{3}$ \\ ${ }^{1}$ NMA/EMBRAPA, Caixa postal 491, 13001-970, Campinas, SP. \\ ${ }^{2}$ Departamento de Estatística/IMEC/UNICAMP, Caixa postal 6560 , \\ 13083-970, Campinas, SP. \\ ${ }^{3}$ Departamento de Zoologia/IB/UNICAMP, Caixa postal 6109, \\ 13081-970, Campinas, SP.
}

An. Soc. Entomol. Brasil 25(2): 191-197 (1996)

Minimum Sample Size of Cotton Plants and Bolls for Monitoring Anthonomus grandis Boh. (Coleoptera: Curculionidae) Populations

\begin{abstract}
Two statistic models were developed to determine the minimum sample size to monitor boll weevil, Anthonomus grandis Boh. (Coleoptera: Curculionidae) populations. The sample consisted of the minimum number of cotton plants and/or bolls surveyed that is needed to detect insect populational densities close to the economic threshold. The crop area was also considered in the monitoring criteria.
\end{abstract}

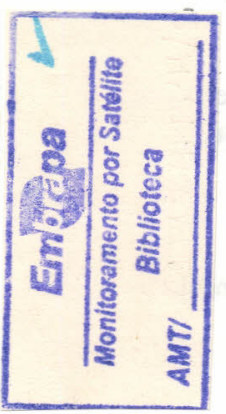

KEY WORDS: Insecta, boll weevil, sampling methods.

RESUMO - Dois modelos estatísticos foram desenvolvidos visando determinar o tamanho mínimo de amostra para monitoramento de populações do bicudo do algodoeiro, Anthonomus grandis Boh. (Coleoptera: Curculionidae). A amostra consistiu no número mínimo de plantas e/ou frutos do algodoeiro avaliados para se detectar as densidades populacionais do inseto próximas ao limiar econômico, considerando-se ainda a área da cultura a ser monitorada.

PALAVRAS-CHAVE: Insecta, bicudo do algodoeiro, métodos de amostragem.
Desde que foi constatada a presença do bicudo do algodoeiro, Anthonomus grandis Boh. no Brasil, vários trabalhos mostraram a viabilidade de utilização de técnicas de manejo, incluindo o emprego de plantas iscas, eliminação dos restos culturais no final do ciclo e utilização de armadilhas com feromônio para controle no período entre safras (Habib et al. 1984, Pierozzi Jr. 1985, 1989, Pierozzi Jr. \& Habib 1992). No entanto, o nível de dano (ND) e o limiar econômico (LE) ainda necessitam ser determinados. Rummel \& Curry (1986) discutem a necessidade de estudos sobre os componentes biológicos, físicos e econômicos envolvidos na interação bicudo-algodoeiro para o estabelecimento de níveis de danos. Pierozzi Jr. (1985) considera 5 a $7 \%$ de botões florais e/ou frutos do algodoeiro atacados como sendo o LE para as lavouras na região de Campinas, SP, nos Estados Unidos o ND varia entre 10 a 15\% (Sartor \& Young 1977).

O bicudo utiliza botões florais e frutos do algodoeiro para sua alimentação e reprodução, deixando sinais nestas estruturas (Pierozzi Jr. \& Habib 1993). O índice de ataque pode ser utilizado como indicador do tamanho da população da praga na 
(8) $n^{\prime}=\frac{t^{2} s^{2}}{d^{2}}$,

onde os mesmos valores de $\mathrm{s}^{2} \mathrm{e} \mathrm{t}$, pois tomouse por base uma amostra conhecida. No entanto, a população de insetos (e a consequente infestação) muda relativamente rápido na lavoura e deseja-se obter um tamanho mínimo para uma nova amostra (n') em uma população onde a variância e a média também mudaram. Assim, a Equação 8 se transforma em:

(9) $n^{\prime}=\frac{t_{1} s_{1}^{2}}{d^{2}}$,

onde d (arbitrário) é previamente fixado. Mas, não se conhece $s_{1}^{2}$ e $t_{1}$, uma vez que o valor de $\mathrm{t}_{1}$ depende do tamanho desconhecido n' da nova amostra. Efetuar o cálculo usando a Equação 9, sem modificação, não seria razoável, pois o que está sendo avaliada é a mudança na população. Uma solução mais geral e rigorosa consiste em por em dúvida tanto $\mathrm{s}_{1}^{2} \mathrm{e} \mathrm{t}_{1}$, conforme Pimentel-Gomes (1990). Tal solução leva à expressão:

(10) $n^{\prime}=\frac{t_{1}{ }^{2} s^{2} F}{d^{2}}, \operatorname{com} F\left(n^{\prime}-1, n-1\right)=\frac{s_{1}{ }^{2}}{s^{2}}$

Esta equação se resolve por tentativa, sem esquecer que $\mathrm{t}_{1}$ é o valor de $\mathrm{t} \operatorname{com}\left(\mathrm{n}^{\prime}-1\right)$ graus de liberdade.

Uma outra solução mais simples, igualmente aceitável e utilizada no presente estudo, é admitir $\mathrm{s}_{1}{ }^{2}=\mathrm{s}^{2}$, onde $\mathrm{s}_{1}{ }^{2}$ é a estimativa da variância na amostra a ser colhida, mas com ( $\left.n^{\prime}-1\right)$ graus de liberdade, obtendo-se finalmente:

(11) $n^{\prime}=\frac{t_{1}^{2} s^{2}}{d^{2}}$,

equação que também se resolve por tentativa, sem esquecer que $t_{1}$ deve ter $\left(n^{\prime}-1\right)$ graus de liberdade.
O segundo modelo, para determinação do número mínimo de frutos a serem coletados e examinados, n", foi calculado conforme as seguintes equações:

(12) $n^{\prime \prime}=\frac{t^{2} p q}{d^{2}+1 / N\left(t^{2} p q-1\right)}$ ou
(13) $n^{\prime \prime}=\frac{t^{2} p q}{d^{2}}$ (quando Né muito grande),

onde: $\mathrm{t}=$ valor percentual da distribuição $\mathrm{t}$ de Student, tirado da tabela bilateral relativo à probabilidade $\alpha ; p=$ proporção amostral de frutos verdes atacados; $q=$ proporção amostral de frutos verdes não atacados; $d=$ definição da Equação 5, considerando-se V(p) = variância da proporção amostral de frutos verdes atacados; $\mathrm{N}=\mathrm{n}^{\circ}$ de frutos verdes na lavoura no dia do monitoramento, considerando-se 50.000 plantas/ha.

Para a validação desses modelos foram utilizados dados do monitoramento semanal, realizado em campos experimentais na região de Campinas, SP $\left(22^{\circ} 35^{\prime}\right.$ e $23^{\circ} 05^{\prime}$ de latitude S e $46^{\circ} 55^{\prime}$ e $47^{\circ} 35^{\prime}$ de longitude W), durante três ciclos do algodão consecutivos a partir de 1984. Este monitoramento consistiu em avaliações de um número variável de plantas onde era determinada a proporção de botões florais e/ou frutos atacados pelo bicudo. Nesta validação, para cada um dos campos estudados, foram considerados apenas os dados referentes às datas de monitoramento nas quais haviam sido detectados valores próximos ao LE considerado, ou seja, 5 a $7 \%$ de botões florais e/ou frutos atacados.

Os dados foram processados através de programas específicos em linguagem Pascal. Os resultados gerados pelos modelos foram valores mínimos do número de plantas ou frutos que poderiam ter sido amostrados, tabelados de acordo com valores de $\alpha$ e d, para cada semana de monitoramento considerada.

No caso da amostragem por conglomerados (infestação avaliada pelo ataque to- 
0,05). O mesmo raciocínio é válido no caso dos frutos verdes. Para os mesmos valores de $\alpha$ e d, os dados obtidos sugerem a avaliação de cerca de 200 frutos/ha. Como o monitoramento deve ser feito semanalmente e os frutos devem ser arrancados da planta, tal amostragem torna-se inviável, neces- sitando- que 350 ha devem ser divididas em áreas menores, as quais devem ser consideradas separadamente em relação ao índice de infestação, aplicando-se o tamanho da amostra para a área de cada divisão. Tal proposição justifica-se pelas seguintes argumentações: (a) devido à dificuldade de se detectar adultos do

Tabela 2. Tamanho mínimo de amostra capaz de detectar o limiar econômico para Anthonomus grandis em campos experimentais durante o ciclo do algodão, 1985-1986.

\begin{tabular}{|c|c|c|c|c|c|c|c|c|c|c|c|c|}
\hline \multicolumn{13}{|c|}{$\begin{array}{c}\text { Campo Experimental } 3(\text { Área }=1,0 \mathrm{ha}) ; \mathrm{np}=200 ; \mathrm{nf}=80 ; \text { botões florais atacados: } 1,3 \% \\
\text { frutos verdes atacados: } 1,3 \%\end{array}$} \\
\hline \multirow[b]{2}{*}{ d } & \multicolumn{2}{|c|}{$\alpha=0,01$} & \multicolumn{2}{|c|}{$\alpha=0,02$} & \multicolumn{2}{|c|}{$\alpha=0,05$} & \multicolumn{2}{|c|}{$\alpha=0,07$} & \multicolumn{2}{|c|}{$\alpha=0,10$} & \multicolumn{2}{|c|}{$\alpha=0,15$} \\
\hline & $\mathrm{n}^{\prime}$ & $\mathrm{n}^{\prime \prime}$ & $\mathrm{n}^{\prime}$ & n" & $\overline{n^{\prime}}$ & n" & $\mathrm{n}^{\prime}$ & $\mathrm{n}^{\prime \prime}$ & $\mathrm{n}^{\prime}$ & $\mathrm{n}^{\prime \prime}$ & $\mathrm{n}^{\prime}$ & n" \\
\hline$\overline{0,01}$ & 21 & 851 & 17 & 694 & 13 & 495 & 6 & 423 & 9 & 347 & 4 & 267 \\
\hline 0,02 & 8 & 217 & 7 & 176 & 5 & 127 & - & 108 & 4 & 89 & - & 68 \\
\hline 0,03 & 6 & 99 & 2 & 80 & - & 57 & - & 49 & - & 40 & - & 31 \\
\hline 0,04 & - & 57 & - & 47 & - & 33 & - & 28 & - & 24 & - & 18 \\
\hline 0,05 & - & 38 & - & 31 & - & 22 & - & 19 & - & 16 & - & 12 \\
\hline 0,07 & - & 21 & - & 17 & - & 13 & - & 11 & - & 9 & - & 7 \\
\hline 0,10 & - & 12 & - & 10 & 8 & - & 7 & - & 6 & - & 5 & \\
\hline
\end{tabular}

Campo Experimental 4 (Área $=3,7 \mathrm{ha}) ; \mathrm{np}=310 ; \mathrm{nf}=65$; botões florais atacados: $2,2 \%$;

frutos verdes atacados: $6,3 \%$



$\mathrm{np}=$ número de plantas e $\mathrm{nf}=$ número de frutos na amostra real; $\mathrm{n}$ = número de plantas e $\mathrm{n}^{\prime \prime}=$ número de frutos gerados pelo modelo.

se estabelecer um número de frutos cuja perda seja suportável para esta finalidade.

Assim, baseando-se nos resultados obtidos na validação dos modelos propõe-se o seguinte plano de amostragem: lavouras com área 1-10 ha: amostra $=100$ plantas e/ou frutos; entre 1150 ha: amostra $=150$; entre 51-150 ha: amostra =200; entre 151-250 ha: amostra $=230$ e entre 251-350 ha: amostra $=250$. Lavouras maiores curculionídeo, principalmente quando em densidades populacionais baixas (Pierozzi Jr. 1989, Rummel \& Curry 1986), utiliza-se a infestação dos sítios de alimentação e reprodução como parâmetro de estimativa do tamanho da população. Enquanto os botões florais podem ser avaliados na planta, os frutos verdes precisam ser arrancados e abertos para avaliação mais segura; (b) o sistema de 
significa assumir que o padrão de ataque de A. grandis numa lavoura de 1 ha seja semelhante àquele que poderá ser observado numa lavoura maior. Para o caso da amostragem dos frutos verdes, segundo as Equações 12 e 13, percebe-se claramente que quando $\mathrm{N}$ é muito grande seu valor não interfere naquele do tamanho de amostra n".

\section{Literatura Citada}

Cochran, N.G. 1965. Técnicas de amostragem. Fundo de Cultura, Rio de Janeiro, RJ. 555p.

Habib, M.E.M., W.D. Fernandes, A. Favaro Jr. \& C.F.S. Andrade. 1984. Eficiência do feromônio de agregação e inseticidas químicos no combate ao bicudo, Anthonomus grandis Boheman. Rev. Agric. 59: 239-251

Pierozzi Jr., I. 1985. Ecologia aplicada de Anthonomus grandis Boh., 1843 (Coleoptera: Curculionidae), na região de Campinas, SP. Tese de mestrado, UNICAMP, Campinas, SP, 155p.

Pierozzi Jr., I. 1989. Análise e aplicabilidade do complexo ecológico de Anthonomus grandis Boheman, 1843 (Coleoptera: Curculionidae), na região de Campinas, SP. Tese de doutorado, UNICAMP, Campinas, SP, 191p.
Pierozzi Jr., I. \& M.E.M. Habib. 1992. Proposta e análise de componentes básicos para um programa de M.I.P. para algodoais infestados por Anthonomus grandis Boheman, 1843 (Coleoptera: Curculionidae), na região de Campinas, SP. Rev. Agric. 67: 253-269.

Pierozzi Jr., I. \& M.E.M. Habib. 1993. Identificação de fatores de mortalidade natural dos estágios imaturos de Anthonomus grandis Boh. (Coleoptera: Curculionidae), na região de Campinas, SP. An. Soc. Entomol. Brasil 22: 325332.

Pimentel-Gomes, F. 1990. Curso de estatística experimental, $13^{\mathrm{a}}$ ed., São Paulo, Livraria Nobel, 468p.

Rummel, D.R. \& Curry, G.L. 1986. Dinâmica populacional e níveis de dano econômico, p. 201-220. In S. Barbosa, M.J. Lukefahr \& R. Braga Sobrinho, (eds.), O bicudo do algodoeiro. Departamento de Difusão de Tecnologia, EMBRAPA, Brasília, DF, 314p.

Sartor, C. \& Young, D. 1977. Cotton scounting manual. Coop. Ext. Serv., Mississippi State University. $N^{\circ} 988$, 20 p.

Recebido em 02/03/95. Aceito em 02/04/96. 